\title{
Supersymmetric Extension of the Sine-Gordon Theory with Integrable Boundary Interactions
}

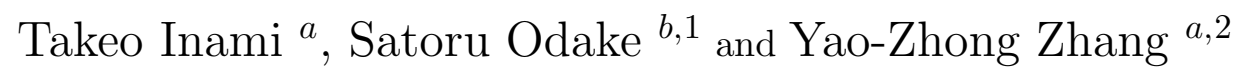 \\ a. Yukawa Institute for Theoretical Physics, Kyoto University, Kyoto 606, Japan \\ b. Department of Physics, Faculty of Science, Shinshu University, Matsumoto 390, Japan
}

\begin{abstract}
Integrability and supersymmetry of the supersymmetric extension of the sine-Gordon theory on a half-line are examined and the boundary potential which preserves both the integrability and supersymmetry on the bulk is derived. It appears that unlike the boundary bosonic sine-Gordon theory, integrability and supersymmetry strongly restrict the form and parameters of the boundary potential, so that no free parameter in the boundary term is allowed up to a choice of signs.
\end{abstract}

\footnotetext{
${ }^{1}$ Email: odake@yukawa.kyoto-u.ac.jp

2 Email: yzzhang@yukawa.kyoto-u.ac.jp; yzz@maths.uq.oz.au.
} 


\section{Introduction}

Integrable quantum field theories in $1+1$ dimensions, both massless and massive, have had interesting applications in particle physics as well as statistical physics near criticality. Many of physical systems in nature have boundaries. In view of applications to such systems, including open string field theory and dissipative quantum mechanics, attempts have recently been made for extending conformal field theories and massive integrable quantum field theories to incorporate boundary interactions, in both field theoretical [1, 2, 3, 4] and algebraic [5, 6] approaches.

A particularly interesting model of massive boundary field theories is the sine-Gordon theory on a half-line [7]. The boundary potential has been obtained and the associated boundary $S$ matrix been constructed by Ghoshal and Zamolodchikov in [3]. One may take the $y$-direction to be the euclidean time and $x$-direction to be the space. The euclidean action of the boundary sine-Gordon theory has been shown to be of the form

$$
S=\int_{-\infty}^{\infty} d y \int_{-\infty}^{0} d x\left\{\frac{1}{2}\left(\partial_{x} \phi\right)^{2}+\frac{1}{2}\left(\partial_{y} \phi\right)^{2}-\frac{m^{2}}{\beta^{2}} \cos \beta \phi\right\}+\left.\Lambda \int_{-\infty}^{\infty} d y \cos \frac{\beta\left(\phi-\phi_{0}\right)}{2}\right|_{x=0},
$$

where $\phi(x, y)$ is a real scalar field, $\beta$ is a dimensionless coupling constant and $m$ is the mass parameter representing the deviation from the massless theory. This model is integrable for arbitrary choices of the parameters $\Lambda$ and $\phi_{0}$ [3]. The same type of analysis has been performed for the (real coupling) affine Toda theories by Corrigan et al [4].

In this paper we study supersymmetric integrable quantum field theories with boundaries. Supersymmetry appears to play a significant role in physics, e.g. superstring field theory, quantum spin-1 chain and surface roughening-reconstruction competition. We consider supersymmetric extension of the sine-Gordon theory on a half-line and derive a boundary potential which preserves both integrability and supersymmetry on the bulk. The bulk supersymmetric sineGordon (SSG) theory can be regarded as integrable deformation of the supersymmetric free field theory (with central charge $c=3 / 2$ ). The boundary conditions for the $c=3 / 2$ theory has previously been studied in the context of open superstring theory [ [ $]$ ]. Only the Neumann boundary condition has been considered, however.

We will first examine the integrability condition and find a few different types of integrable boundary terms. Supersymmetry is broken in general in a theory with boundaries unless suitable boundary conditions are chosen. We will derive a general form of boundary potential which restores (one half of) supersymmetry. We will then compare the result from integrability with that from supersymmetry.

\section{SSG Theory on the Bulk}

The euclidean action of the SSG theory on the whole line $(-\infty, \infty)$ or on the bulk is given by

$$
S_{0}=\int d x d y \mathcal{L}_{0}
$$




$$
\begin{aligned}
\mathcal{L}_{0}= & \frac{1}{2}\left(\partial_{x} \phi\right)^{2}+\frac{1}{2}\left(\partial_{y} \phi\right)^{2}-\bar{\psi}\left(\partial_{x}-i \partial_{y}\right) \bar{\psi}+\psi\left(\partial_{x}+i \partial_{y}\right) \psi \\
& -\frac{m^{2}}{\beta^{2}} \cos \beta \phi-2 m \bar{\psi} \psi \cos \frac{\beta \phi}{2},
\end{aligned}
$$

where $\psi, \bar{\psi}$ are the two components of a Majorana fermion.

We use the notation: $z=x+i y, \bar{z}=x-i y$. The classical equations of motion on the bulk are

$$
\begin{aligned}
& \partial_{\bar{z}} \partial_{z} \phi=\frac{m^{2}}{4 \beta} \sin \beta \phi+\frac{m \beta}{4} \bar{\psi} \psi \sin \frac{\beta \phi}{2}, \\
& \partial_{\bar{z}} \psi=-\frac{m}{2} \bar{\psi} \cos \frac{\beta \phi}{2}, \\
& \partial_{z} \bar{\psi}=-\frac{m}{2} \psi \cos \frac{\beta \phi}{2} .
\end{aligned}
$$

The SSG theory on the whole line is integrable. It is the simplest of supersymmetric affine Toda theory, and is known to be associated with the superalgebra $o s p(2 \mid 2)^{(2)}$. An infinite number of conserved charges has been derived at the classical level [9] and the quantum level [10].

The supersymmetry of the model is manifest if we write the above component expression in terms of superfield:

$$
S_{0}=\int d^{2} z d^{2} \theta\left\{\frac{1}{2} \bar{D} \Phi D \Phi-\frac{2 m}{\beta^{2}} \cos \frac{\beta \Phi}{2}\right\},
$$

where $\bar{D}$ and $D$ are covariant derivatives in the superspace coordinates $\bar{z}, \bar{\theta}$ and $z, \theta$, respectively,

$$
\bar{D}=-\partial_{\bar{\theta}}+\bar{\theta} \partial_{\bar{z}}, \quad D=\partial_{\theta}+\theta \partial_{z}
$$

and $\Phi$ is a (scalar) superfield,

$$
\Phi=\phi+i \theta \psi-i \bar{\theta} \bar{\psi}+i \bar{\theta} \theta F
$$

with $F$ an auxiliary field. The equation of motion in terms of the superfield reads

$$
\bar{D} D \Phi=\frac{m}{\beta} \sin \frac{\beta \Phi}{2} .
$$

\section{SSG Theory on a Half-Line}

The theory on a half-line $x \in(-\infty, 0]$ is defined by adding the boundary term to the bulk part $S_{0}$ of the action,

$$
S=S_{0}+S_{b} \equiv \int_{-\infty}^{\infty} d y \int_{-\infty}^{0} d x \mathcal{L}_{0}+\int_{-\infty}^{\infty} d y \mathcal{B}(\phi, \psi, \bar{\psi})
$$

where the boundary potential $\mathcal{B}$ is assumed to be a functional of the fields at $x=0$ but not their derivatives. In addition to the bulk field equation (2.2), we have equations of motion at the boundary $x=0$,

$$
\begin{aligned}
\partial_{x} \phi+\frac{\partial \mathcal{B}}{\partial \phi} & =0 \\
\psi-\frac{\partial \mathcal{B}}{\partial \psi} & =0 \\
\bar{\psi}+\frac{\partial \mathcal{B}}{\partial \bar{\psi}} & =0
\end{aligned}
$$


From this, we remark that at $x=0, \partial^{2} \mathcal{B} / \partial \phi \partial \psi=\partial^{2} \mathcal{B} / \partial \phi \partial \bar{\psi}=\partial^{2} \mathcal{B} / \partial \psi \partial \bar{\psi}=0$.

For simplicity we rescale the fields $\phi, \psi$ and $\bar{\psi}$ in the following way: $\phi \rightarrow \phi / \beta, \psi \rightarrow$ $\psi / \beta, \bar{\psi} \rightarrow \bar{\psi} / \beta$, and restrict our attention to the classical case $\beta \rightarrow 0$. Moreover we set $m=2$ so that the bulk equations of motion simplify to the form

$$
\begin{aligned}
& \partial_{\bar{z}} \partial_{z} \phi=\sin \phi+\frac{1}{2} \bar{\psi} \psi \sin \frac{\phi}{2}, \\
& \partial_{\bar{z}} \psi=-\bar{\psi} \cos \frac{\phi}{2} \\
& \partial_{z} \bar{\psi}=-\psi \cos \frac{\phi}{2}
\end{aligned}
$$

As is known [9, 10], in the bulk theory there is an infinite number of conserved charges constructed from densities $T_{s+1}, \bar{T}_{s+1}, \Theta_{s-1}$ and $\bar{\Theta}_{s-1}$ with $s=1,3,5, \cdots$. These densities satisfy the following continuity equations

$$
\partial_{\bar{z}} T_{s+1}=\partial_{z} \Theta_{s-1}, \quad \partial_{z} \bar{T}_{s+1}=\partial_{\bar{z}} \bar{\Theta}_{s-1} .
$$

The densities of $s=1$ are given by the energy-momentum tensor,

$$
\begin{aligned}
& T_{2}=\left(\partial_{z} \phi\right)^{2}-\partial_{z} \psi \psi \\
& \bar{T}_{2}=\left(\partial_{\bar{z}} \phi\right)^{2}+\partial_{\bar{z}} \bar{\psi} \bar{\psi}, \\
& \Theta_{0}=\bar{\Theta}_{0}=-2 \cos \phi-\bar{\psi} \psi \cos \frac{\phi}{2} .
\end{aligned}
$$

The $s=3$ densities are [9, 10

$$
\begin{aligned}
T_{4} & =\left(\partial_{z}^{2} \phi\right)^{2}-\frac{1}{4}\left(\partial_{z} \phi\right)^{4}+\frac{3}{4}\left(\partial_{z} \phi\right)^{2} \partial_{z} \psi \psi-\partial_{z}^{2} \psi \partial_{z} \psi \\
\bar{T}_{4} & =\left(\partial_{\bar{z}}^{2} \phi\right)^{2}-\frac{1}{4}\left(\partial_{\bar{z}} \phi\right)^{4}-\frac{3}{4}\left(\partial_{\bar{z}} \phi\right)^{2} \partial_{\bar{z}} \bar{\psi} \bar{\psi}+\partial_{\bar{z}}^{2} \bar{\psi} \partial_{\bar{z}} \bar{\psi} \\
\Theta_{2} & =\left(\partial_{z} \phi\right)^{2} \cos \phi-\partial_{z} \psi \psi \cos ^{2} \frac{\phi}{2}-\bar{\psi} \partial_{z} \psi \partial_{z} \cos \frac{\phi}{2}+\frac{1}{4} \bar{\psi} \psi\left(\partial_{z} \phi\right)^{2} \cos \frac{\phi}{2} \\
\bar{\Theta}_{2} & =\left(\partial_{\bar{z}} \phi\right)^{2} \cos \phi+\partial_{\bar{z}} \bar{\psi} \bar{\psi} \cos ^{2} \frac{\phi}{2}+\psi \partial_{\bar{z}} \bar{\psi} \partial_{\bar{z}} \cos \frac{\phi}{2}+\frac{1}{4} \bar{\psi} \psi\left(\partial_{\bar{z}} \phi\right)^{2} \cos \frac{\phi}{2}
\end{aligned}
$$

Suppose that one can choose boundary potential such that at $x=0$

$$
T_{4}-\bar{T}_{4}-\left(\Theta_{2}-\bar{\Theta}_{2}\right)=\frac{d}{d y} \Sigma_{3}(y)
$$

where $\Sigma_{3}(y)$ is some functional of boundary fields. Then the charge $P_{3}$, given by

$$
P_{3}=\int_{-\infty}^{0} d x\left(T_{4}+\bar{T}_{4}+\Theta_{2}+\bar{\Theta}_{2}\right)-i \Sigma_{3}(y)
$$

is a non-trivial integral of motion.

We now examine in what circumstances $T_{4}-\bar{T}_{4}-\left(\Theta_{2}-\bar{\Theta}_{2}\right)$ may be written as a total $y$-derivative. Observing that

$$
\psi_{x x}=4 \psi \cos ^{2} \frac{\phi}{2}-2 i \bar{\psi}_{y} \cos \frac{\phi}{2}-2 \bar{\psi} \partial_{x} \cos \frac{\phi}{2}-i \psi_{x y}
$$




$$
\begin{aligned}
\bar{\psi}_{x x} & =4 \bar{\psi} \cos ^{2} \frac{\phi}{2}+2 i \psi_{y} \cos \frac{\phi}{2}-2 \psi \partial_{x} \cos \frac{\phi}{2}+i \bar{\psi}_{x y} \\
\psi_{x y} & =-2 \bar{\psi}_{y} \cos \frac{\phi}{2}-2 \bar{\psi} \partial_{y} \cos \frac{\phi}{2}-i \psi_{y y} \\
\bar{\psi}_{x y} & =-2 \psi_{y} \cos \frac{\phi}{2}-2 \psi \partial_{y} \cos \frac{\phi}{2}+i \bar{\psi}_{y y}
\end{aligned}
$$

one can show that

$$
\begin{aligned}
T_{4}-\bar{T}_{4}-\left(\Theta_{2}-\bar{\Theta}_{2}\right)= & -\frac{i}{2}\left(\phi_{x x}-\phi_{y y}\right) \phi_{x y}+\frac{i}{8}\left(\phi_{x}^{2}-\phi_{y}^{2}\right) \phi_{x} \phi_{y}+i \phi_{x} \phi_{y} \cos \phi \\
& +\frac{3 i}{16}\left(\phi_{x}^{2}-\phi_{y}^{2}\right)\left(\bar{\psi}_{y} \bar{\psi}-\psi_{y} \psi\right)+i 3 \cos ^{2} \frac{\phi}{2}\left(\bar{\psi}_{y} \bar{\psi}-\psi_{y} \psi\right) \\
& -i \partial_{x} \cos \frac{\phi}{2}\left(\bar{\psi}_{y} \psi-\psi_{y} \bar{\psi}\right)+i\left(\bar{\psi}_{y y} \bar{\psi}_{y}-\psi_{y y} \psi_{y}\right) \\
& +i \bar{\psi} \psi \phi_{x} \phi_{y} \cos \frac{\phi}{2}-\frac{3}{8} \phi_{x} \phi_{y}\left(\psi_{y} \psi+\bar{\psi}_{y} \bar{\psi}\right) \\
& +2 \partial_{y} \cos \frac{\phi}{2}\left(\bar{\psi}_{y} \psi-\bar{\psi} \psi_{y}\right)+\cos \frac{\phi}{2}\left(\bar{\psi} \psi_{y y}-\bar{\psi}_{y y} \psi\right),
\end{aligned}
$$

where $Q_{x}, Q_{x y}$ etc stand for $\partial_{x} Q, \partial_{x} \partial_{y} Q$ and so on. If one notices that

$$
\begin{aligned}
& \phi_{x x}=4 \sin \phi-\phi_{y y}+2 \bar{\psi} \psi \sin \frac{\phi}{2} \\
& \phi_{x y}=-\frac{\partial^{2} \mathcal{B}}{\partial \phi^{2}} \phi_{y}-\frac{\partial^{2} \mathcal{B}}{\partial \psi \partial \phi} \psi_{y}-\frac{\partial^{2} \mathcal{B}}{\partial \bar{\psi} \partial \phi} \bar{\psi}_{y}=-\frac{\partial^{2} \mathcal{B}}{\partial \phi^{2}} \phi_{y},
\end{aligned}
$$

eq.(3.10) can be written as

$$
\begin{aligned}
T_{4}+\bar{\Theta}_{2}-\bar{T}_{4}-\Theta_{2}= & \text { ("bosonic part") }+i \bar{\psi} \psi \phi_{y}\left(\frac{\partial^{2} \mathcal{B}}{\partial \phi^{2}} \sin \frac{\phi}{2}-\frac{\partial \mathcal{B}}{\partial \phi} \cos \frac{\phi}{2}\right) \\
& +\frac{3 i}{16}\left(\left(\frac{\partial \mathcal{B}}{\partial \phi}\right)^{2}-\phi_{y}^{2}+16 \cos ^{2} \frac{\phi}{2}\right)\left(\bar{\psi}_{y} \bar{\psi}-\psi_{y} \psi\right) \\
& -i \partial_{x} \cos \frac{\phi}{2}\left(\bar{\psi}_{y} \psi-\psi_{y} \bar{\psi}\right)+i\left(\bar{\psi}_{y y} \bar{\psi}_{y}-\psi_{y y} \psi_{y}\right) \\
& +\frac{3}{8} \frac{\partial \mathcal{B}}{\partial \phi} \phi_{y}\left(\psi_{y} \psi+\bar{\psi}_{y} \bar{\psi}\right) \\
& +2 \partial_{y} \cos \frac{\phi}{2}\left(\bar{\psi}_{y} \psi-\bar{\psi} \psi_{y}\right)+\cos \frac{\phi}{2}\left(\bar{\psi} \psi_{y y}-\bar{\psi}_{y y} \psi\right),
\end{aligned}
$$

where the "bosonic part" is given by

$$
\begin{aligned}
-i \frac{\partial^{2} \mathcal{B}}{\partial \phi^{2}} \phi_{y y} \phi_{y}+\frac{i}{8} \frac{\partial \mathcal{B}}{\partial \phi} \phi_{y}^{3}+i\left(-\frac{1}{8}\left(\frac{\partial \mathcal{B}}{\partial \phi}\right)^{3}+2 \frac{\partial^{2} \mathcal{B}}{\partial \phi^{2}} \sin \phi-\frac{\partial \mathcal{B}}{\partial \phi} \cos \phi\right) \phi_{y} \\
=i \partial_{y}\left(\frac{1}{8} \mathcal{B} \phi_{y}^{2}+\int^{\phi}\left(-\frac{1}{8}\left(\frac{\partial \mathcal{B}}{\partial \phi}\right)^{3}+2 \frac{\partial^{2} \mathcal{B}}{\partial \phi^{2}} \sin \phi-\frac{\partial \mathcal{B}}{\partial \phi} \cos \phi\right) d \phi\right) \\
-i\left(\frac{\partial^{2} \mathcal{B}}{\partial \phi^{2}}+\frac{1}{4} \mathcal{B}\right) \phi_{y} \phi_{y y}+\frac{i}{8} \phi_{y}^{2}\left(\bar{\psi}_{y} \bar{\psi}-\psi_{y} \psi\right) .
\end{aligned}
$$

Taking account of the result in the bosonic case [3], we look for the solution $\mathcal{B}(\phi, \psi, \bar{\psi})$ of the form

$$
\mathcal{B}(\phi, \psi, \bar{\psi})=\mathcal{B}_{b}(\phi)+\mathcal{B}_{f}(\psi, \bar{\psi})
$$


where $\mathcal{B}_{b}$ is the boundary potential in the bosonic sine-Gordon theory: $\mathcal{B}_{b}=\Lambda \cos \frac{\phi-\phi_{0}}{2}$ with $\Lambda$ and $\phi_{0}$ being arbitrary constants; $\mathcal{B}_{f}$ is a function of the fields $\psi$ and $\bar{\psi}$. The purely bosonic part in (3.12) is then a total $y$-derivative, and hence we only have to deal with the remaining bilinear terms in $\psi$ and $\bar{\psi}$. $\mathcal{B}_{f}$ can be written as

$$
\mathcal{B}_{f}(\psi, \bar{\psi})=M \bar{\psi} \psi+\epsilon \psi+\bar{\epsilon} \bar{\psi}
$$

with $M$ being a bosonic parameter and $\epsilon, \bar{\epsilon}$ constant fermionic parameters. Then the equations of motion at boundary $x=0$ become

$$
\begin{aligned}
& \text { 1) } \quad \psi=-\frac{\epsilon+M \bar{\epsilon}}{1-M^{2}}, \quad \bar{\psi}=\frac{\bar{\epsilon}+M \epsilon}{1-M^{2}} \quad \text { for } M \neq \pm 1 \text {, } \\
& \text { 2) } \bar{\psi}=\mp(\psi+\epsilon), \quad \bar{\epsilon}=\mp \epsilon \quad \text { for } M= \pm 1 \text {. }
\end{aligned}
$$

It is not difficult to see, after some exercise, that we have two solutions in order for the r.h.s. of (3.12) to be a total $y$-derivative

$$
\begin{aligned}
& \text { 1) } \quad M \neq \pm 1, \Lambda, \phi_{0}, \epsilon, \bar{\epsilon} \text { arbitrary; } \\
& \text { 2) } \quad M= \pm 1, \quad \Lambda= \pm 8, \quad \phi_{0}=0, \quad \bar{\epsilon}=0=\epsilon .
\end{aligned}
$$

Returning to the original normalization, one has two different forms of boundary potential compatible with the integrability on the bulk:

$$
\begin{aligned}
\text { 1) } \mathcal{B}(\phi, \psi, \bar{\psi}) & =\Lambda \cos \frac{\beta\left(\phi-\phi_{0}\right)}{2}+M \bar{\psi} \psi+\epsilon \psi+\bar{\epsilon} \bar{\psi}, \quad(M \neq \pm 1) \\
\text { 2) } \mathcal{B}(\phi, \psi, \bar{\psi}) & = \pm \frac{4 m}{\beta^{2}} \cos \frac{\beta \phi}{2} \pm \bar{\psi} \psi .
\end{aligned}
$$

These equations give rise to the following boundary conditions at $x=0$, respectively,

$$
\begin{aligned}
\text { 1) } \partial_{x} \phi & =\frac{\beta \Lambda}{2} \sin \frac{\beta\left(\phi-\phi_{0}\right)}{2}, \quad \psi=-\frac{\epsilon+M \bar{\epsilon}}{1-M^{2}}, \quad \bar{\psi}=\frac{\bar{\epsilon}+M \epsilon}{1-M^{2}}, \quad(M \neq \pm 1) ; \\
\text { 2) } \partial_{x} \phi & = \pm \frac{2 m}{\beta} \sin \frac{\beta \phi}{2}, \quad \psi \pm \bar{\psi}=0 .
\end{aligned}
$$

These boundary potentials have been derived by examining the first non-trivial conserved charge $P_{3}$, where we have restricted attention to the classical case. We believe that our analysis will be completed by showing that all conserved charges of higher spin give the same results, and that the above computation can be extended to the quantum theory by taking into account the normal ordering.

\section{Supersymmetric Boundary Interactions on the Half-Line}

Again we work with $(3.3)$ and $\mathcal{L}_{0}\left(\mathcal{S}_{0}\right)$ corresponding to it. Supersymmetry transformation is given by

$$
\begin{aligned}
\delta_{s} \phi & =\eta \psi+\bar{\eta} \bar{\psi}, \\
\delta_{s} \psi & =-\eta \partial_{z} \phi-2 \bar{\eta} \sin \frac{\phi}{2}, \\
\delta_{s} \bar{\psi} & =\bar{\eta} \partial_{\bar{z}} \phi+2 \eta \sin \frac{\phi}{2},
\end{aligned}
$$


where $\eta, \bar{\eta}$ are fermionic parameters. It can be checked that under the transformation (4.1), $\mathcal{L}_{0}$ changes by a total derivative:

$$
\begin{aligned}
\delta_{s} \mathcal{L}_{0}= & \partial_{x}\left\{\frac{1}{2} \partial_{x} \phi(\eta \psi+\bar{\eta} \bar{\psi})-2 \sin \frac{\phi}{2}(\eta \bar{\psi}+\bar{\eta} \psi)+\frac{i}{2} \partial_{y} \phi(\eta \psi-\bar{\eta} \bar{\psi})\right\} \\
& +\partial_{y}\left\{\frac{1}{2} \partial_{y} \phi(\eta \psi+\bar{\eta} \bar{\psi})+i 2 \sin \frac{\phi}{2}(\eta \bar{\psi}-\bar{\eta} \psi)-\frac{i}{2} \partial_{x} \phi(\eta \psi-\bar{\eta} \bar{\psi})\right\} .
\end{aligned}
$$

It follows immediately that the theory defined by the lagrangian $\mathcal{L}_{0}$ is supersymmetric on the whole line. However, this is not true for the theory on the half-line, because the boundary destroys the supersymmetry of the action. In fact, (4.2) implies that

$$
\delta_{s} S_{0}=\left.\int_{-\infty}^{\infty} d y\left\{\frac{1}{2} \partial_{x} \phi(\eta \psi+\bar{\eta} \bar{\psi})-2 \sin \frac{\phi}{2}(\eta \bar{\psi}+\bar{\eta} \psi)+\frac{i}{2} \partial_{y} \phi(\eta \psi-\bar{\eta} \bar{\psi})\right\}\right|_{x=0} .
$$

The r.h.s. of the above equation is non-zero.

In order to preserve supersymmetry for the theory on the half-line one may either add a boundary term to the action or impose boundary condition by hand on $\phi, \psi$ and $\bar{\psi}$, in such a way as to cancel the total $x$-derivative terms in (4.2).

The boundary potential which restores supersymmetry is obtained by solving the equation

$$
\delta_{s} S_{0}+\delta_{s} S_{\mathcal{B}}=0
$$

On dimensional consideration, we take the candidate for $\mathcal{B}$ to be of the form

$$
\mathcal{B}=\Lambda_{s} \cos \frac{\phi-\phi_{s}}{2}+M_{s} \bar{\psi} \psi
$$

where $\Lambda_{s}, \phi_{s}$ and $M_{s}$ are constant bosonic parameters. Then

$$
\begin{aligned}
\delta_{s} S_{\mathcal{B}}= & \int_{-\infty}^{\infty} d y\left\{-\frac{\Lambda_{s}}{2} \sin \frac{\phi-\phi_{s}}{2} \delta_{s} \phi+M_{s} \delta_{s} \bar{\psi} \psi+M_{s} \bar{\psi} \delta_{s} \psi\right\} \\
= & \int_{-\infty}^{\infty} d y\left\{\left(-\frac{\Lambda_{s}}{2} \sin \frac{\phi-\phi_{s}}{2}+2 M_{s} \sin \frac{\phi}{2}\right)(\eta \psi+\bar{\eta} \bar{\psi})\right. \\
& \left.+\frac{M_{s}}{2} \partial_{x} \phi(\bar{\eta} \psi+\eta \bar{\psi})+\frac{i M_{s}}{2} \partial_{y} \phi(\bar{\eta} \psi-\eta \bar{\psi})\right\} .
\end{aligned}
$$

It turns out that there is no solution unless

$$
\bar{\eta}=\mp \eta
$$

The fact that only one of $\eta$ and $\bar{\eta}$ is independent implies that only half of the supersymmetry on the bulk is preserved on the half-line. One encounters the same situation in the case of open superstring theory [8].

Restricting to the choice (4.7), we have found two solutions:

$$
M_{s}= \pm 1, \quad \phi_{s}=0, \quad \Lambda_{s}= \pm 8 .
$$

Therefore the boundary term which cancels the total derivative of the lagrangian $\mathcal{L}_{0}$ is given by

$$
\mathcal{B}= \pm\left(8 \cos \frac{\phi}{2}+\bar{\psi} \psi\right)
$$


The existence of two solutions corresponding to the choice of two kinds of half supersymmetry (4.7) is reminiscent of two models of superstring, Neveu-Schwarz and Ramond.

It remains to examine whether the boundary potential (4.9) has a room for other terms. Such terms, if they exist, must be invariant under the (half-) supersymmetry transformation. Allowing for fermionic parameters, we propose the following term to be added to the boundary potential (4.9): $\epsilon_{s} \psi+\bar{\epsilon}_{s} \bar{\psi}$. Under the supersymmetry transformation, it transforms as

$$
\epsilon_{s} \delta_{s} \psi+\bar{\epsilon}_{s} \delta_{s} \bar{\psi}=-\frac{1}{2} \partial_{x} \phi\left(\epsilon_{s} \pm \bar{\epsilon}_{s}\right) \eta+2 \sin \frac{\phi}{2}\left(\bar{\epsilon}_{s} \pm \epsilon_{s}\right) \eta+i \frac{1}{2} \partial_{y} \phi\left(\epsilon_{s} \mp \bar{\epsilon}_{s}\right) \eta .
$$

We find that (4.10) is a $y$-derivative if

$$
\bar{\epsilon}_{s}=\mp \epsilon_{s}
$$

This implies that the term $\epsilon_{s}(\psi \mp \bar{\psi})$ is left invariant under the half supersymmetry transformation and therefore can be freely added to the boundary potential (4.9).

Returning to the original normalization, we have the boundary potential which preserves the supersymmetry on the bulk:

$$
\mathcal{B}= \pm \frac{4 m}{\beta^{2}} \cos \frac{\beta \phi}{2} \pm \bar{\psi} \psi+\epsilon_{s}(\psi \mp \bar{\psi})
$$

which leads to the following boundary condition at $x=0$ :

$$
\begin{aligned}
& \partial_{x} \phi= \pm \frac{2 m}{\beta} \sin \frac{\beta \phi}{2}, \\
& \psi \pm \bar{\psi}=-\epsilon_{s} .
\end{aligned}
$$

This boundary condition is supersymmetry preserving, i.e. for $\bar{\eta}=\mp \eta$ we have $\delta_{s}\left(\partial_{x} \phi \mp 4 \sin \frac{\phi}{2}\right)=$ $-i \eta \partial_{y}\left(\psi \pm \bar{\psi}+\epsilon_{s}\right)=0$ and $\delta_{s}\left(\psi \pm \bar{\psi}+\epsilon_{s}\right)=-\eta\left(\partial_{x} \phi \mp 4 \sin \frac{\phi}{2}\right)=0$.

Let us examine the possibility of imposing boundary condition by hand. It is easily seen that for arbitrary parameters $\eta$ and $\bar{\eta}$ there is no nontrivial choice of boundary condition for the r.h.s. of eq.(4.3) to be vanishing. However if one sets $\bar{\eta}=\mp \eta$ in (4.3), then

$$
\delta_{s} S_{0}=\left.\int_{-\infty}^{\infty} d y\left\{\left(\frac{1}{2} \partial_{x} \phi \pm 2 \sin \frac{\phi}{2}\right) \eta(\psi \mp \bar{\psi})+\frac{i}{2} \partial_{y} \phi \eta(\psi \pm \bar{\psi})\right\}\right|_{x=0}
$$

and one may have two choices of boundary conditions at $x=0$ :

$$
\begin{aligned}
\text { i) } & \partial_{x} \phi=\mp 4 \sin \frac{\phi}{2}, \quad \psi \pm \bar{\psi}=0 ; \\
\text { ii) } & \partial_{y} \phi=0, \quad \psi \mp \bar{\psi}=0 .
\end{aligned}
$$

We want to see if these boundary conditions imposed by hand are left invariant by the supersymmetry transformation $\delta_{s}$ with the parameters $\eta, \bar{\eta}$ satisfying $\bar{\eta}=\mp \eta$. It is easy to check that (4.16) is supersymmetric invariant whereas 4.15) is not.

Both the boundary conditions (4.15) and (4.16) do not coincide with that in section 3 . Therefore they are not compatible with the integrability on the half-line. 


\section{Integrability vs. Supersymmetry: Conclusion}

As can be seen from sections 3 and 4 , only when one chooses $\epsilon_{s}=0=\bar{\epsilon}_{s}$, are (4.12) and (4.13) derived from supersymmetry consideration compatible with (3.21) and (3.23) derived from integrability consideration. Therefore the total euclidean action which preserves both integrability and supersymmetry on the bulk is

$$
S=\int_{-\infty}^{\infty} d y \int_{\infty}^{0} d x \mathcal{L}_{0}+\left.\int_{-\infty}^{\infty} d y\left\{ \pm \frac{4 m}{\beta^{2}} \cos \frac{\beta \phi}{2} \pm \bar{\psi} \psi\right\}\right|_{x=0}
$$

and the boundary condition at $x=0$ is

$$
\partial_{x} \phi= \pm \frac{2 m}{\beta} \sin \frac{\beta \phi}{2}, \quad \psi \pm \bar{\psi}=0 .
$$

To summarize, we have constructed the boundary potential for the supersymmetric extension of the sine-Gordon theory on the half-line by imposing both integrability and supersymmetry. The potential thus obtained is unique modulo overall sign. In addition there is a class of boundary potential (3.20) which preserves integrability but breaks supersymmetry and another class (4.12) which preserves supersymmetry but is not integrable.

We would like to thank K. Kobayashi, R. Sasaki, K. Schoutens and T. Uematsu for discussions. This work was partially supported by the Grant-in-Aid for Scientific Research from the Ministry of Education, Science and Culture of Japan. Y.Z.Z. is supported by the Kyoto University Foundation.

\section{References}

[1] I. Cherednik, Theor.Math.Phys. 61 (1984) 35.

[2] J. Cardy, Nucl.Phys. B324 (1989) 581.

[3] S. Ghoshal, A.B. Zamolodchikov, Int.J.Mod.Phys. A9 (1994) 3841.

[4] E. Corrigan, P.E. Dorey, R.H. Rietdijk, R. Sasaki, Phys.Lett. B333 (1994) 83.

[5] E.K. Sklyanin, J.Phys. A: Math.Gen. 21 (1988) 2375.

[6] L. Mezincescu, R.I. Nepomechie, J.Phys. A: Math.Gen. 24 (1991) L17.

[7] E.K. Sklyanin, Funct.Anal.Appl. 21 (1987) 164.

[8] P. Di Vecchia, B. Durhuus, P. Olesen, J.L. Petersen, Nucl.Phys. B207 (1982) 77.

[9] L. Girardello, S. Sciuto, Phys.Lett. B77 (1978) 267.

[10] R. Sasaki, I. Yamanaka, Prog.Theor.Phys. 79 (1988) 1167. 